\title{
Measurement of Crystal Thickness and Crystal Tilt From HRTEM Images and a Way to Correct for Their Effects
}

\author{
SVEN HOVMÖLLER* AND XIAODONG ZOU \\ Structural Chemistry, Stockholm University, S-106 91 Stockholm, Sweden
}

\author{
KEY WORDS image restoration; electron microscopy; structure determination; crystallographic \\ structure factors; amplitudes and phases; thickness determination
}

\begin{abstract}
The effects of thickness and tilt angle are studied numerically on experimental high-resolution transmission electron microscope (HRTEM) images of a wedge-shaped metal oxide crystal. For sufficiently thin and well-aligned crystals, the amplitudes and phases of the Fourier transforms of the HRTEM images are essentially the same as the crystallographic structurefactors. F or tilted crystals, the changes of amplitudes and phases as a function of increased thickness and tilt angle can be described by a simple model. A method is presented by which the local thickness can be determined from one HRTEM image and one convergent-beam el ectron diffraction pattern from the same crystal. It is also shown how the projected potential can be reconstructed from HRTEM images of tilted crystals, discl osing the crystal structure, even from quite thick ( $>20 \mathrm{~nm}$ ) samples. Microsc. Res. Tech. 46:00-00, 1999. ๑1999 Wiley-Liss, Inc.
\end{abstract}

\section{INTRODUCTION}

Modern 200-400 kV high resolution transmission electron microscopes (HRTEM) have a resolution of between 0.25 and $0.15 \mathrm{~nm}$. This is sufficient for resolving all non-hydrogen atoms, since the interatomic distances are in the range 1.5-2.5 $\AA$. In some favorable cases, such as metal oxides or al loys with one short unit cell dimension $(<5 \AA)$, a single projection along the short axis may reveal the positions of all metal atoms. A resolution of $0.25 \mathrm{~nm}$ is sufficient for locating the metal atoms in oxides within $0.1 \AA$ (Hovmöller et al., 1984), while about $0.17 \mathrm{~nm}$ resolution is needed for locating all atoms in alloys and other metal-rich compounds (Weirich et al., 1996). For more complex structures, it may be necessary to do a full three-dimensional reconstruction of the structure, as shown by Downing et al., 1990.

In practice, a direct interpretation of HRTEM images in terms of crystal structures is only possible if all experimental conditions are close to optimal. These include microscope alignment including beam tilt (Saxton and Smith, 1985), crystal thickness, defocus value, crystal orientation, and others. If one or more of these requirements is not fulfilled, image processing has to be applied before the image can be interpreted. The effects of beam tilt are not studied in this paper, but it is assumed that beam tilt is so small that it is negligible. This is probably a realistic assumption in most cases.

Several schemes of reconstruction of out-of-focus images have been devised in order to determine the projected potential, or at least the image of what it would have been under ideal experimental conditions, allowing a direct interpretation in terms of atomic positions. Erickson and Klug (1971) showed how the contrast transfer function (CTF) varies with defocus, how the defocus value can be obtained from the Thon rings in the Fourier transform (FT) of HRTEM images, and finally described how an undistorted image could be reconstructed by correcting for the CTF by computerized image processing. The method of Erickson and
Klug has recently been implemented in the image processing program CRISP (Hovmöller, 1992) and applied successfully to HRTEM images of inorganic crystals by Zou et al. (1996). The defocus value can be determined also by other methods (Li, 1998; Van Dyck et al., 1996).

The effects of crystal thickness on HRTEM images have been extensively studied using image simulations. In spite of the importance of sample thickness for the appearance of HRTEM images, there has hitherto not existed any generally applicable and straightforward method to determine this parameter. Instead, a series of simulated images is typically calculated, within a range of thickness that presumably includes that of the experimental area (Spence and Cowley, 1995). J ansen et al. (1998) have developed a method for obtaining a value of the crystal thickness in small areas by combining multi-slice calculations and least-squares refinement of a structure model against electron diffraction data.

Crystal tilt has a great effect on HRTEM images. It may sometimes be an advantage to take images of slightly tilted crystals, since these have a smoother projected potential and thus are less affected by multiple scattering, which otherwise distorts the images such that they are no longer proportional to the projected potential. A slight tilt may reduce the dynamical scattering by as much as a factor of 2 (O'Keefe and Radmilovic, 1993). Crystal tilt is a major reason why HRTEM images of thin crystals often cannot be interpreted directly. Thus, it is important to look closer at the effects of crystal tilt and to find a method for correcting HRTEM images that are affected by crystal

\footnotetext{
Contract grant sponsor: Swedish Natural Science Research Council (NFR). *Correspondenceto: Sven Hovmöller, Structural Chemistry, Stockholm University, S-106 91 Stockholm Sweden. E-mail: svenh@struc.su.se

Received 9 March 1998; accepted in revised form 12 F ebruary 1999
} 
tilt. This is so much more important, since only rarely are image simulations carried out with crystal tilts included. In a previous study on simulated images of the silicate mineral orthopyroxene, Zou et al. (1995) showed that crystallographic image processing (CIP) could be used to reconstruct interpretable images of tilted crystals. Here a similar approach is taken for compensating for the effects of crystal tilt on experimental HRTEM images.

The main effect of crystal tilt is to smear out the structural information in the direction perpendicular to the tilt axis. This lowers the resolution, but only in that direction. F or thin crystals and small tilt angles, where the weak phase object approximation is valid, the effects of crystal tilt are proportional to the tilt angle multiplied by the crystal thickness. For small tilts, the effects are mainly an attenuation of the amplitudes of the image, while phases are unaffected. These effects were derived in theory by Zou (1995) and are here demonstrated quantitatively on experimental HRTEM images of a wedge-shaped crystal .

The traditional way of interpreting HRTEM images, by matching with image simulations, is typically carried out by calculating a matrix of some five different defocus values and five different crystal thicknesses, while keeping astigmatism, beam tilt, and crystal tilt at zero. If these three latter parameters were also all owed to take on different values in the image simulations, they would each require about a 10-fold increase in the number of images calculated. This large number arises since astigmatism, beam tilt, and crystal tilt each need two numbers to describe them; a direction and a magnitude. Clearly it is not practically possible to simulate tens of thousands of images for each structure model. We propose an al ternative approach (apart from the obvi ous experimental efforts to keep all these error parameters as close to zero as possible); to detect and quantify each of these experimental parameters. If we know the crystal thickness, defocus, tilt, etc. , then there is no need to simulate more than a single image, which then has to fit with the experimental one. Before image simulations can be applied to an unknown structure, it must first be solved, for example from the projected potential reconstructed from HRTEM images by image processing, after correcting for the various distortions.

We present here a method for determination of crystal thickness and tilt angle in HRTEM images. The method can be used both on uniformly thick and wedge-shaped crystals. We al so show that the projected potential can be faithfully reconstructed even from thick, tilted crystals simply by imposing the crystal symmetry.

\section{MATERIALS AND METHODS Electron Microscopy}

HRTEM images and electron diffraction patterns of $\mathrm{K}_{2} \mathrm{O} \cdot 7 \mathrm{Nb}_{2} \mathrm{O}_{5}$ were taken in a J EOL (Tokyo, J apan) 4000 electron microscope operated at $400 \mathrm{kV}$, with a magnification of 400,000 on Kodak (Rochester, NY) $\mathrm{SO}-163 \mathrm{Film} . \mathrm{K}_{2} \mathrm{O} \cdot 7 \mathrm{~N} \mathrm{~b}_{2} \mathrm{O}_{5}$ is tetragonal, $\mathrm{P} 4 / \mathrm{mbm}$ with unit cell dimensions $a=b=27.5 \AA$ and $c=3.94 \AA$. The structure of this compound has been solved by electron crystallography (Hu et al., 1992), that of the isostructural thallium compound by X-ray crystallography
(Bhide and Gasparin, 1979), and the cesium analog by electron crystallography (Wang et al., 1988).

An electron transparent wedge-shaped crystal was sel ected and al igned to have the short c-axis parallel to the electron beam. A series of images was taken from the same area of the crystal, with nominal tilt angles ranging from 0 to 5 degrees. At each tilt angle, electron diffraction patterns were taken from the same area, by using both selected area electron diffraction (SAED) and convergent beam electron diffraction (CBED).

\section{Image Digitization}

The photographic negatives were put on a light box and digitized, using a DAGE (Michigan City, I L) MTI CCD72 video-rate CCD camera with 8 bit grey-scale resolution, connected to a Shark II + frame grabber. The 256 grey levels provided by 8 bits is sufficient for digitizing HRTEM images (but not for SAED patterns). Similarly, the non-linearity of photographic film is not a problem here. Parts of the HRTEM images that all corresponded to the same area, near the edge of the crystal, were digitized. The digitization was done with $768 \times 576$ pixels, each $24 \times 24 \mu \mathrm{m}$, corresponding to $0.06 \times 0.06 \mathrm{~nm}$ per pixel. This is sufficient for recovering all structural information out to at least $0.15 \mathrm{~nm}$ resol ution, since an oversampling of at least 2 is needed to follow a periodic feature. The processed area was about $45 \times 35 \mathrm{~nm}^{2}$, or just over 16 by 12 unit cells (Fig. 1).

\section{Determination of Tilt Angle}

The tilt axis was roughly perpendicular to the edge of the crystal (see Fig. 1). The directions and magnitudes of the tilts were determined manually from the CBED patterns, by finding the center of the Laue circle, as seen in Figure 2. The tilt angle was then calculated from the geometry shown in Figure 3. It must be emphasized that this method leads to the same result, independent of where on the crystal the convergent electron beam is placed, provided of course that the crystal is not bent.

The direction of thetilt axis can also be seen in the FT of an HRTEM image, from the line of strong diffraction spots (Fig. 4). However, the magnitude of the tilt (the tilt angle) cannot be determined from a single $\mathrm{FT}$ since the attenuation of diffraction spots away from the tilt axis depends on the tilt angle multiplied by the crystal thickness. At least two HRTEM images from the same area, with a known increment in tilt angle between them would be needed for separating the effects of thickness and tilt.

\section{I mage Analysis}

The HRTEM images were processed by F ourier techniques using the Windows version of the imageprocessing program CRI SP (H ovmöller, 1992). FTs were calculated from either the entire digitized area (Fig. 5) or from selected bands with varying crystal thickness (Fig. 4).

The image amplitudes and phases can easily be extracted from the FT of the images (Unwin and Henderson, 1975) and then used directly to obtain the crystallographic structure factors, which are the ones that must be known in order to solve a crystal struc- 


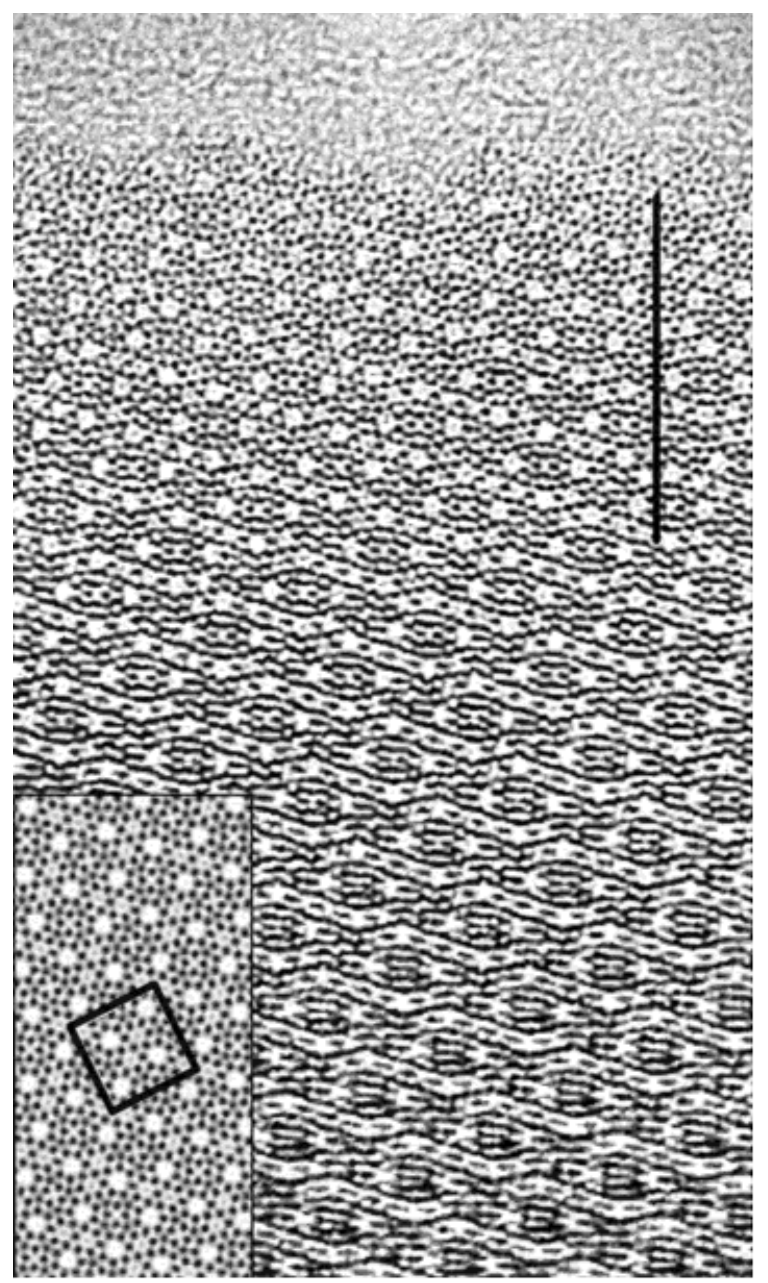

Tilt angle:

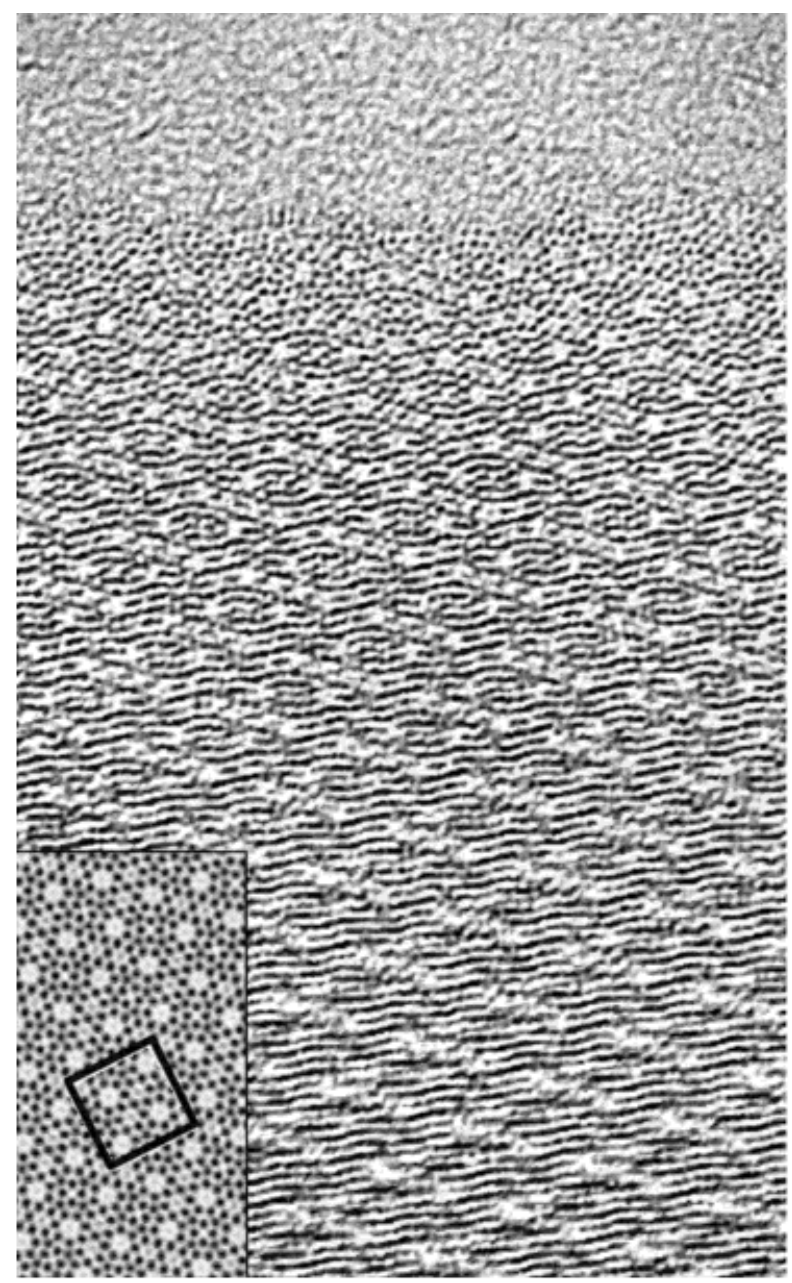

$2.5^{\circ}$

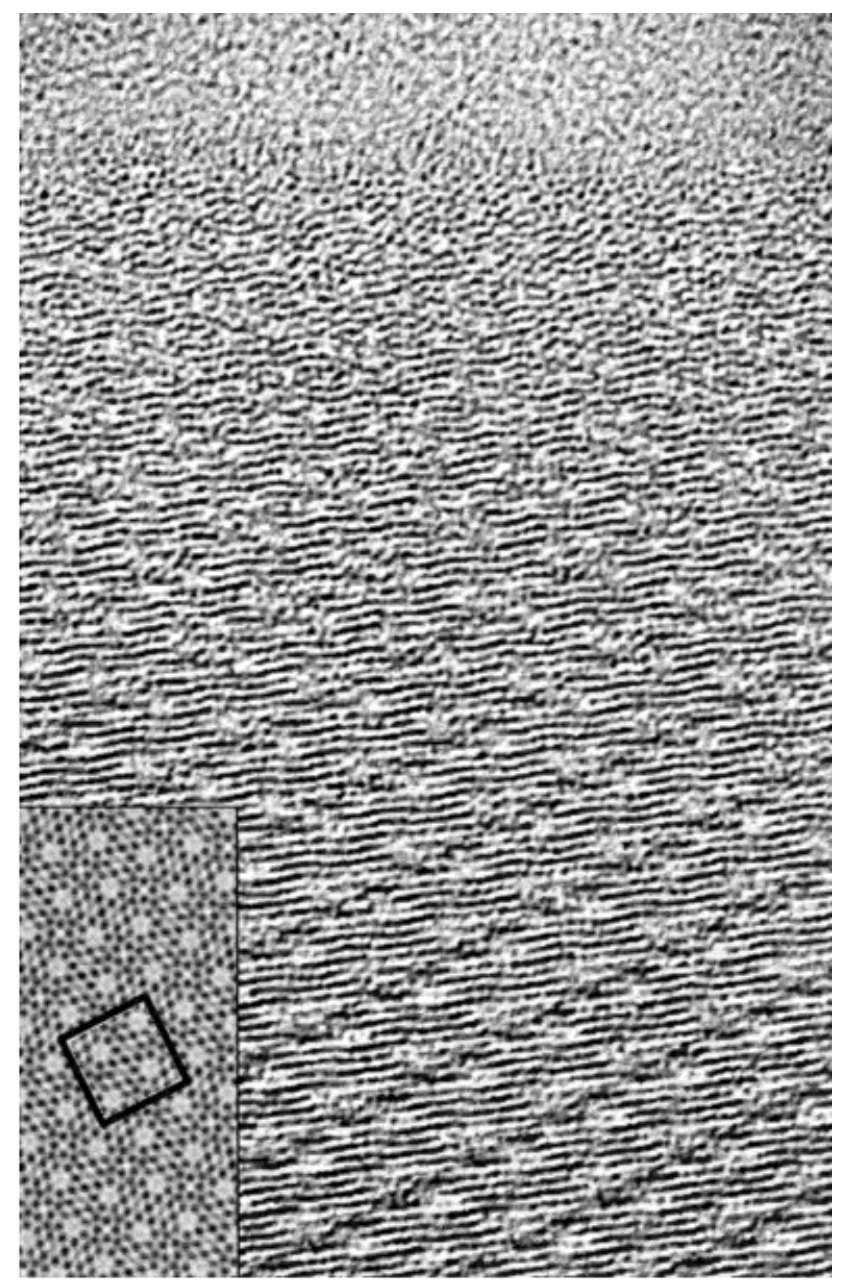

$4.1^{\circ}$

Fig. 1. HRTEM images of the same area of a wedge-shaped crystal of $\mathrm{K}_{2} \mathrm{O} \cdot 7 \mathrm{Nb}_{2} \mathrm{O}_{5}$, tilted to various degrees. The tilt axis is nearly vertical, i.e., perpendicular to the edge of the crystal. Insets: Reconstructed projected potentias after crystallographic image processing. The unit
cell $(27.5 \AA \times 27.5 \AA)$ is drawn in the insets. The vertical scale bar is $10 \mathrm{~nm}$ long. The effect of

crystal tilt is a smearing of the potential in the direction perpendicular to the tilt axis. This is seen as the nearly horizontal stripes that become increasingly pronounced with higher tilt angles and thicker crystal. 


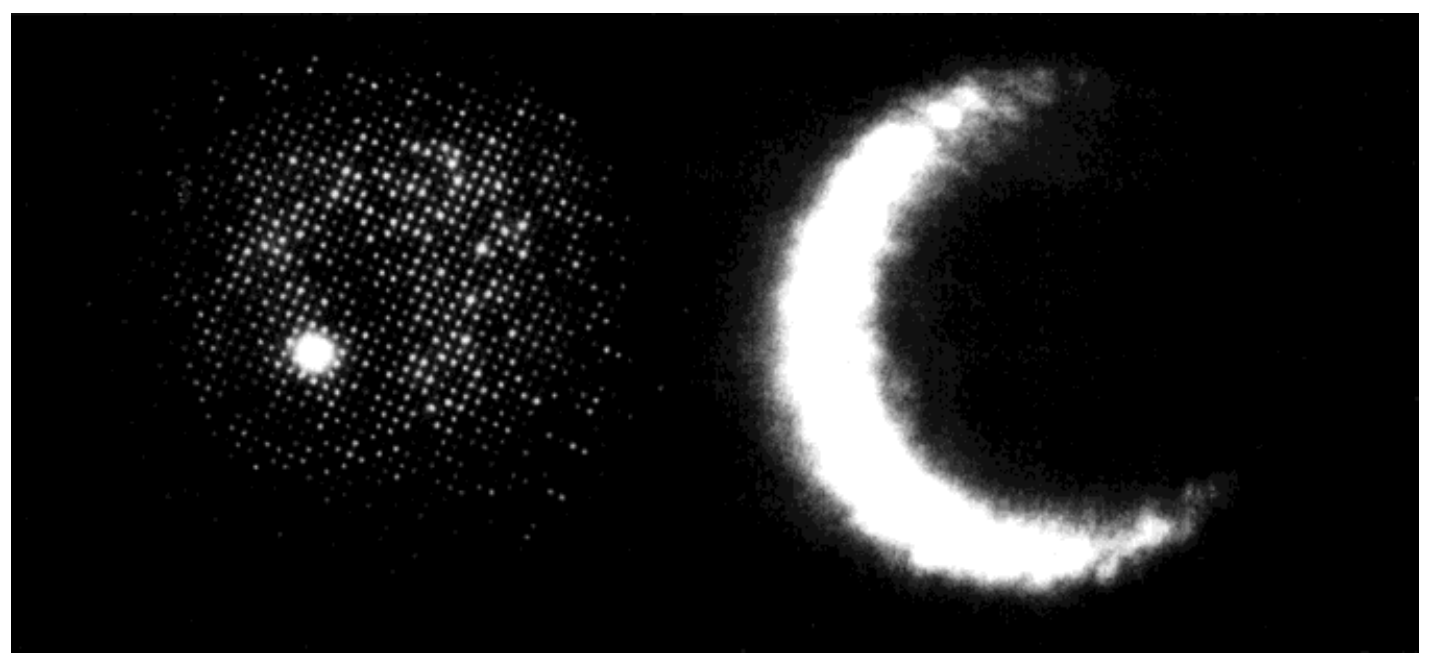

Fig. 2. SAED (left) and CBED (right) patterns of $\mathrm{K}_{2} \mathrm{O} \cdot 7 \mathrm{Nb}_{2} \mathrm{O}_{5}$ tilted $0.35^{\circ}$ and $1.10^{\circ}$, respectively. The SAED pattern was used to calibrate the scale of the el ectron diffraction patterns, while the CBED patterns were used for finding the center of the E wald spheres for each tilt angle. In the diffraction patterns above the projections are along $(7,7,0)$ (left) and $(6,16,0)$ (right), with tilt angles of $0.34^{\circ}$ and $1.1^{\circ}$ respectively.

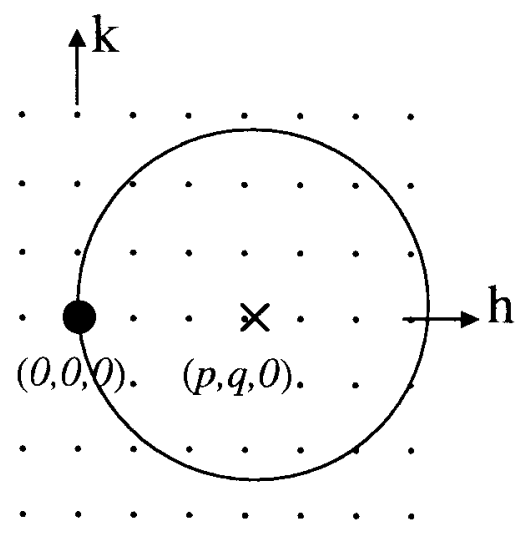

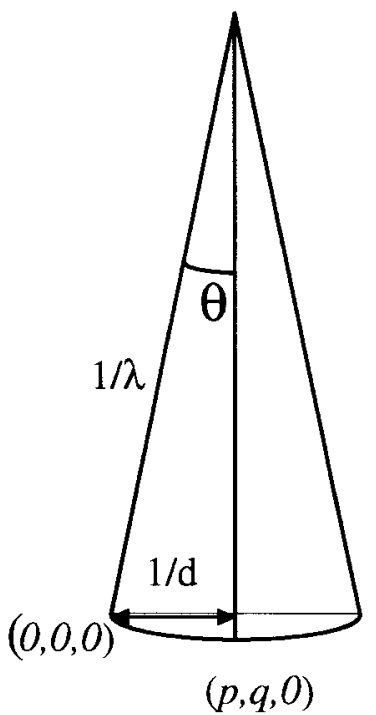

Fig. 3. Geometrical estimation of tilt angle Top (left) and side view (right). The tilt angle $\theta$ is calculated from $\sin \theta=\lambda / d$, where $\lambda$ is the wavelength of the electron and $d$ is the $d$-value of position $(p, q, 0)$. ture. The phases of the FT of an image of a perfectly aligned crystal of a structure with a centrosymmetric projection remain close to $0^{\circ}$ or $180^{\circ}$, and so can only be correct or wrong by $180^{\circ}$. The phase errors caused by random noise and systematic errors, such as multiple diffraction, are typically within $20^{\circ}$ in good images of thin areas.

Throughout this paper, the word phase is used in the sense of crystallographic structure factor phases, either as their values in the crystals or as those experimentally measured estimates of the structure factor phases that we obtain from the FT of the HRTEM images.

Qualitatively, the effects of crystal tilt and thickness were investigated by calculating so-called quasi-optically filtered images in the foll owing way. From the FT of the whole digitized area, just one single diffraction spot and its Friedel mate were selected, using the
Lattice Filter option in CRISP. The inverse FT of this diffraction pair was calculated. In this way, the amplitude and phase of a single diffraction spot could be followed visually over the entire area of the scanned crystal, as seen in Figure 5.

Quantitatively, these effects were investigated in several ways, all in Fourier space. In the quasi-optically filtered images, the amplitude and phase of a single reflection was measured at regular intervals, along a line perpendicular to the edge of the crystal. The plots of a few of these reflections are shown in Figure 6.

The effects of crystal tilt as a function of tilt angleand crystal thickness were investigated quantitatively al so by processing a set of narrow bands ( 4 by $30 \mathrm{~nm}$ ) of the crystal at different distances from the crystal edge, and thus of increasing thickness. The bands had their long direction parallel to the edge of the crystal. For each 

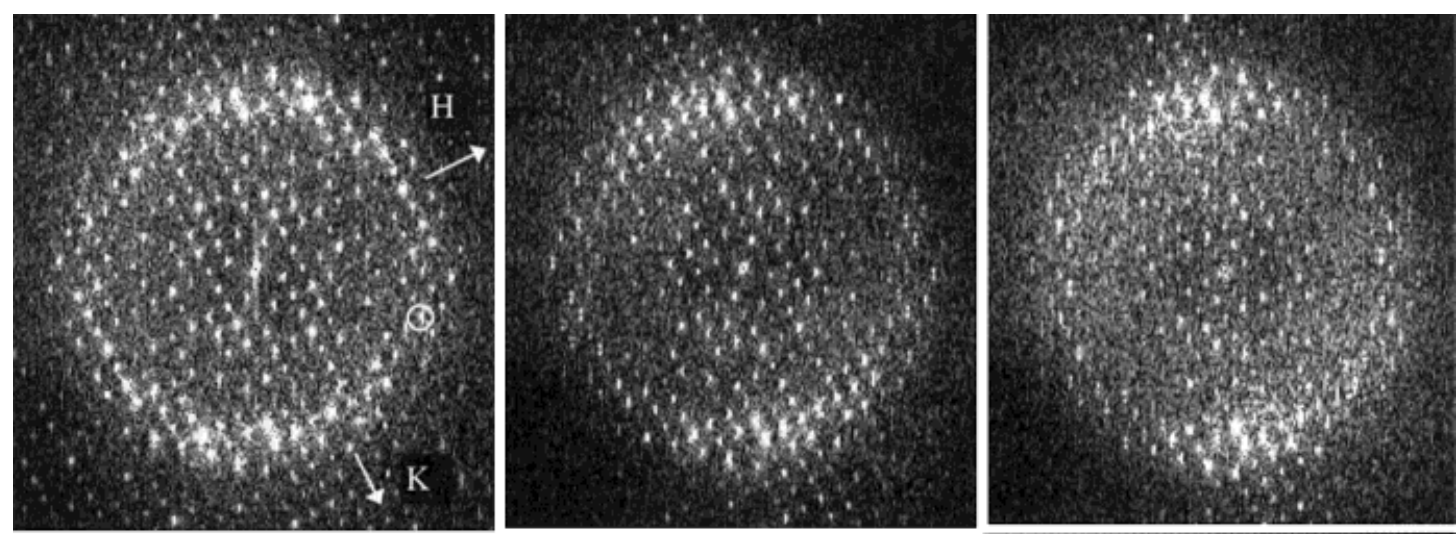

Distance
from edge
of crystal
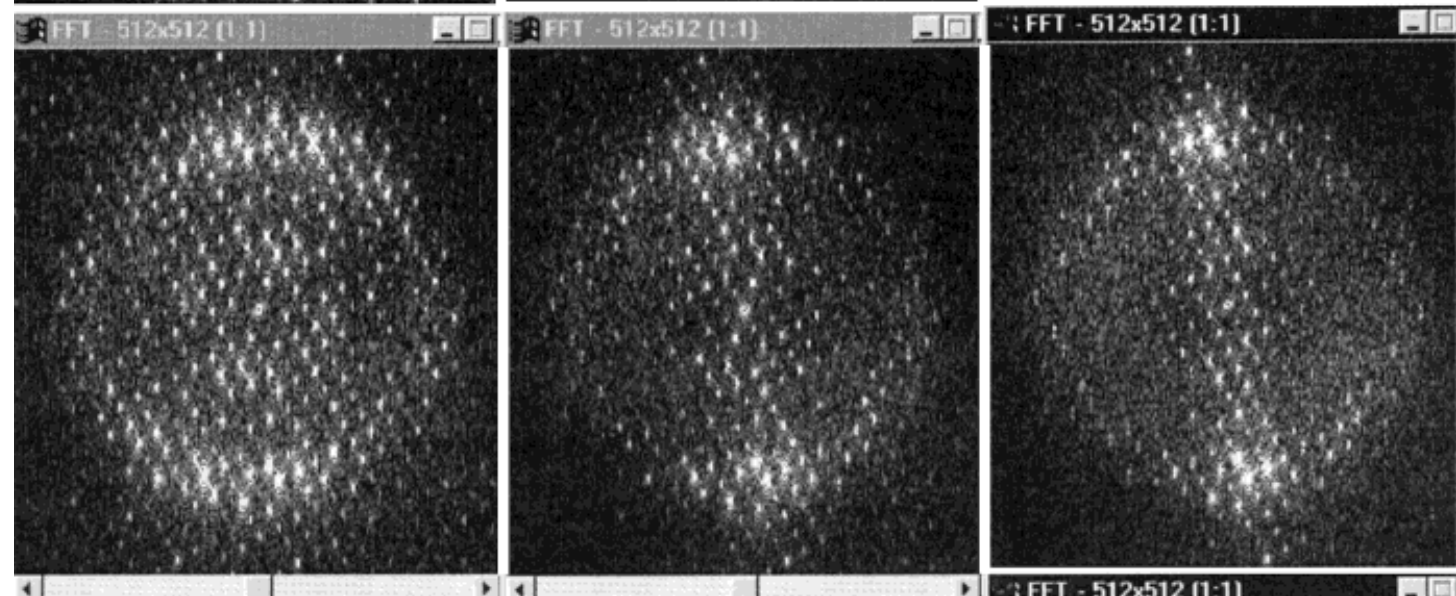

0-120 ̊
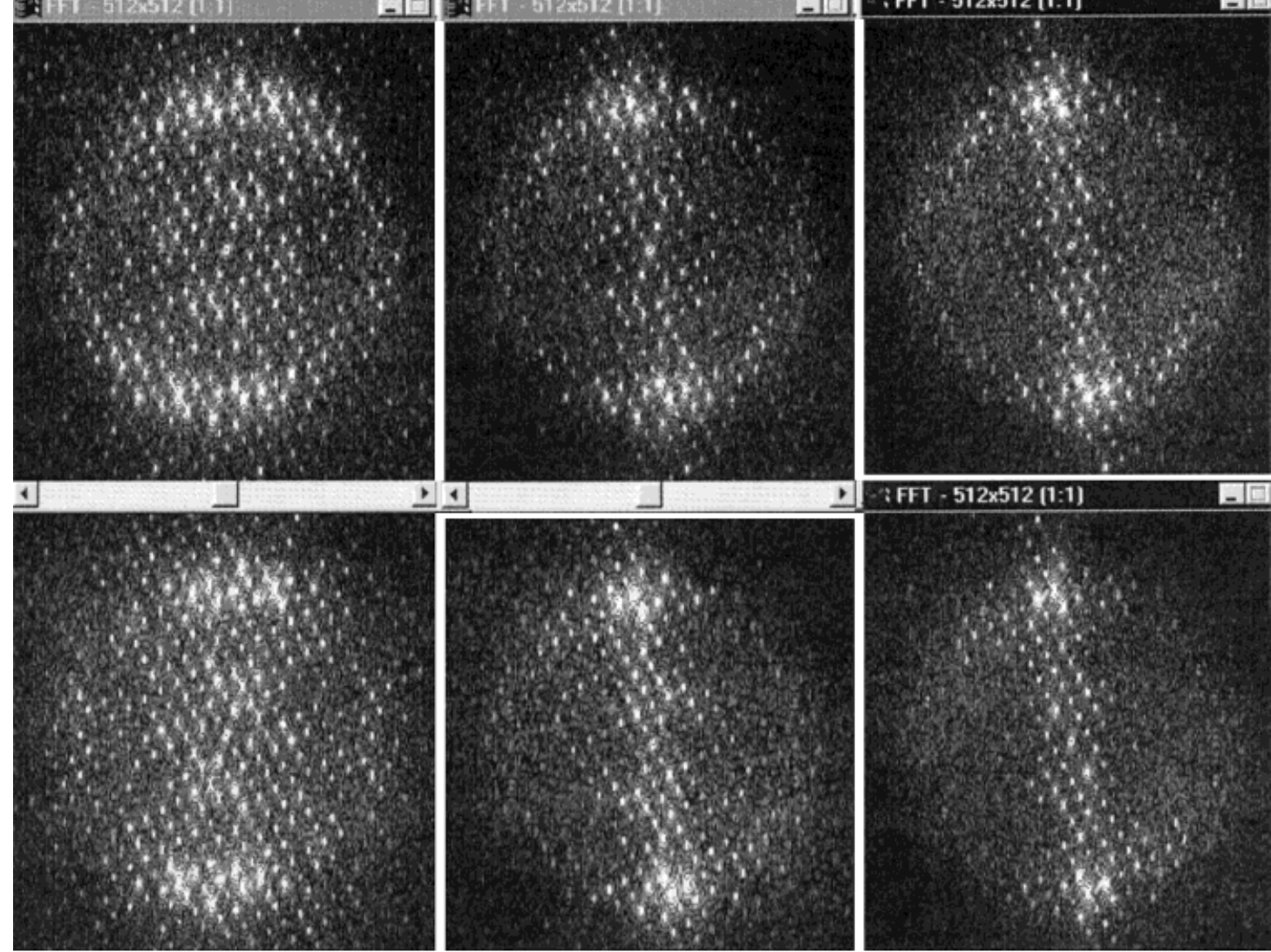

120-240 Å

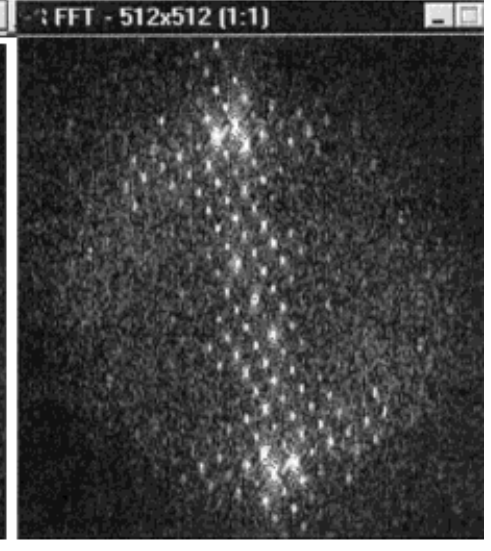

240-360 ̊

\section{Tilt angle:}

$1.1^{\circ}$ $2.5^{\circ}$

$4.1^{\circ}$

Fig. 4. Calculated Fourier transforms of parts of thethree HRTEM images. The tilt axis is vertical. It can be seen that the effects of increasing tilt angle (going right in the figure) and increasing the crystal thickness (going down in the figure) are quite similar. In both cases, the reflections on the tilt axis are unaffected, while the

band, the FT was calculated and the lattice of diffraction spots in the calculated diffractogram was indexed. All diffractograms were indexed the same way, with the $h$ axis running roughly parallel to the edge of the crystal and the k axis nearly parallel to the tilt axis. The amplitudes and crystallographic structure factor phases of the indexed diffraction spots within a resolu- reflections away from the tilt axis decrease in amplitude as a function of (tilt angle) $\times$ (crystal thickness) $\times$ (distance of reflection from the tilt axis). The crystallographic axes $\mathrm{H}$ and $\mathrm{K}$ are indicated with arrows. The reflection $(6,6)$, marked with a ring, was selected for quasi-optical filtering, as seen in Figure 5.

tion of $0.17 \mathrm{~nm}$ were extracted from the FT. The phase origin of each band was then shifted, such that they all coincided with the same (of the two possible) 4-fold axis within the unit cell. Amplitudes and phases of all the reflections were compared numerically in order to see how they were affected by crystal tilt and crystal thickness. The amplitudes and phases for four of the 


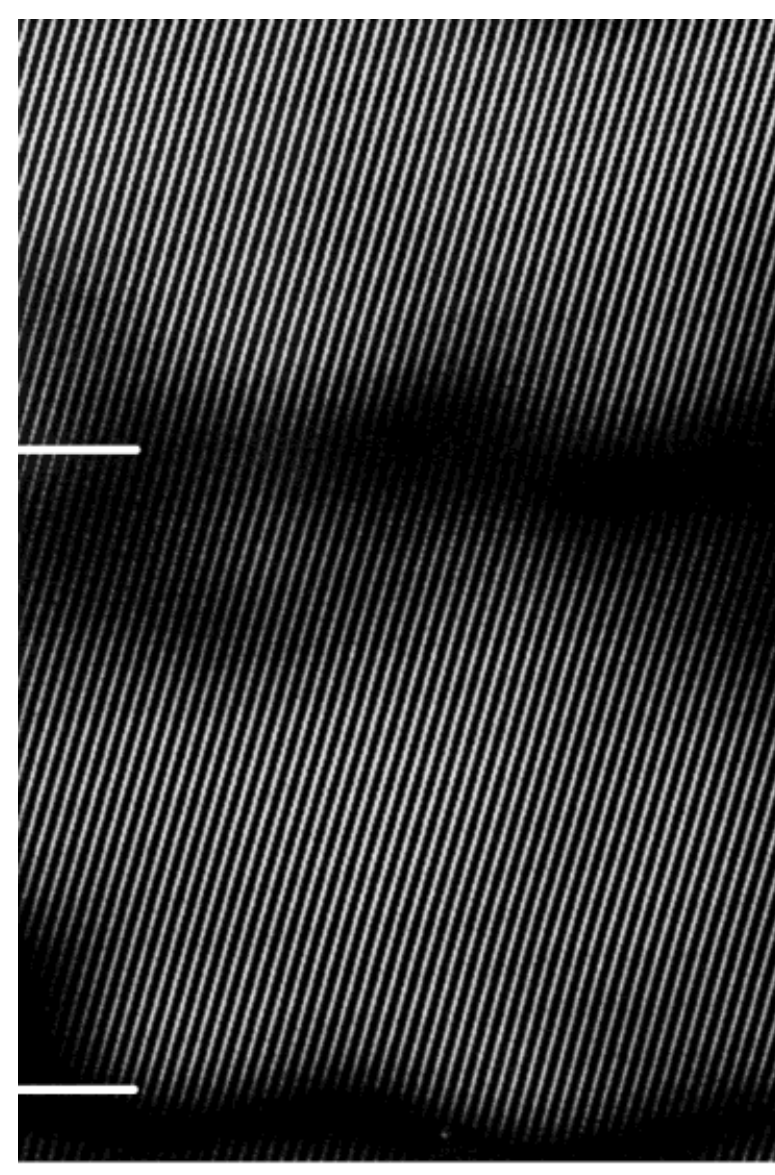

Tilt angle:

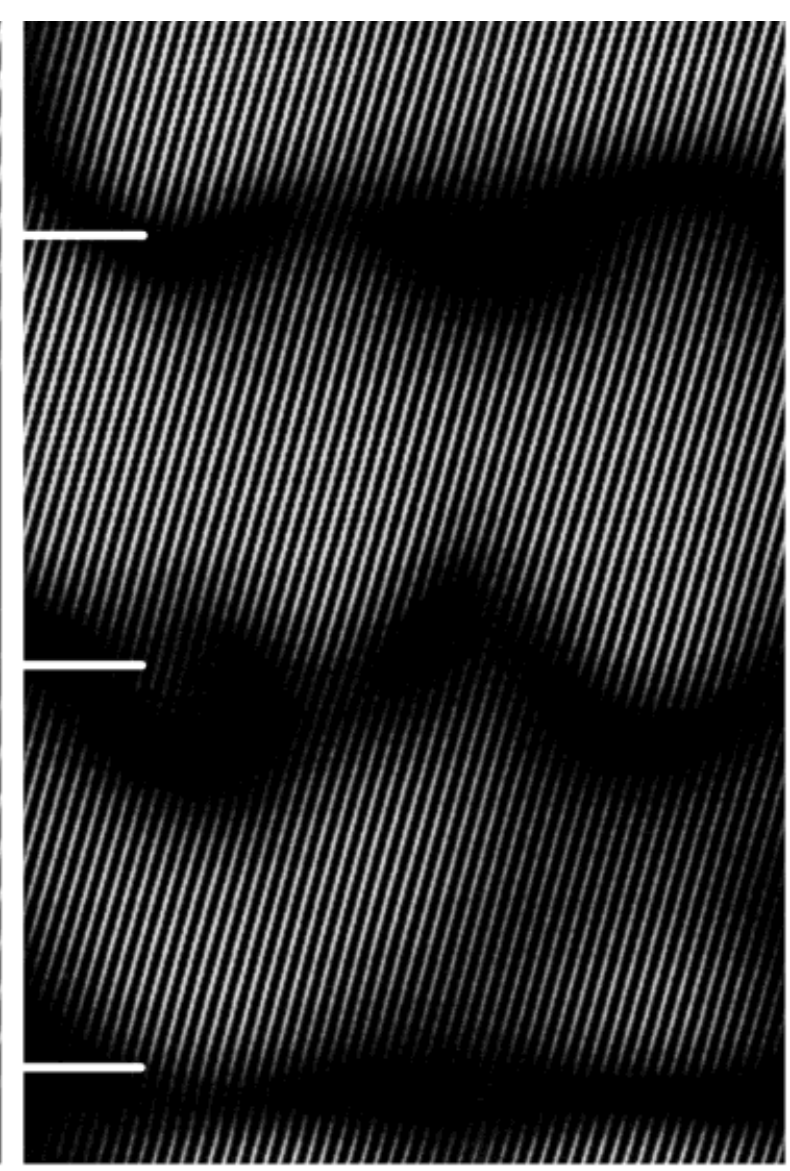

$2.5^{\circ}$

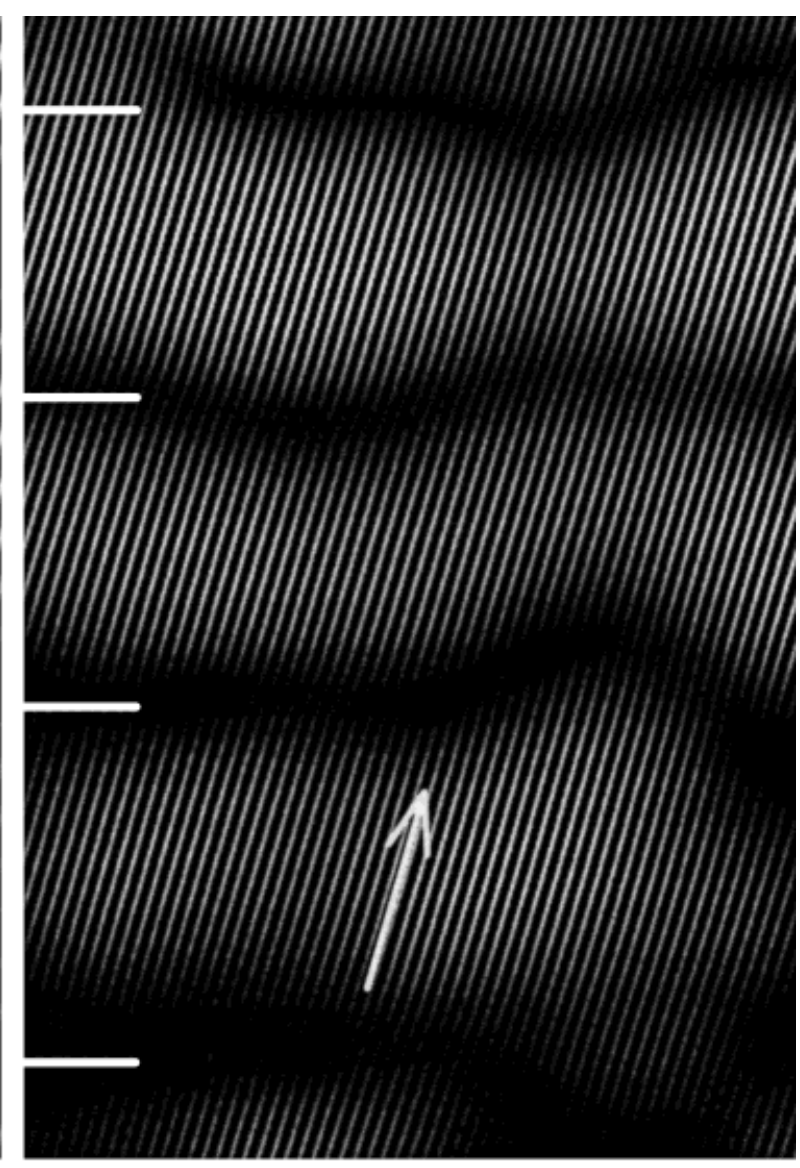

4.1 ${ }^{\circ}$

Fig. 5. Quasi-optical filtering, showing the phase and amplitude of the reflection (6 6). The three panels are all from the same area of the crystal, but the tilt angle differs. The images show 2, 3, and 4 phase cross-overs, respectively (marked with white lines). The phase of the reflection shifts by $180^{\circ}$ (i.e., the the contrast reverses) on either side of a cross-over. This is best seen by looking at an oblique angle along the arrow, with the eye al most in the plane of the paper. 


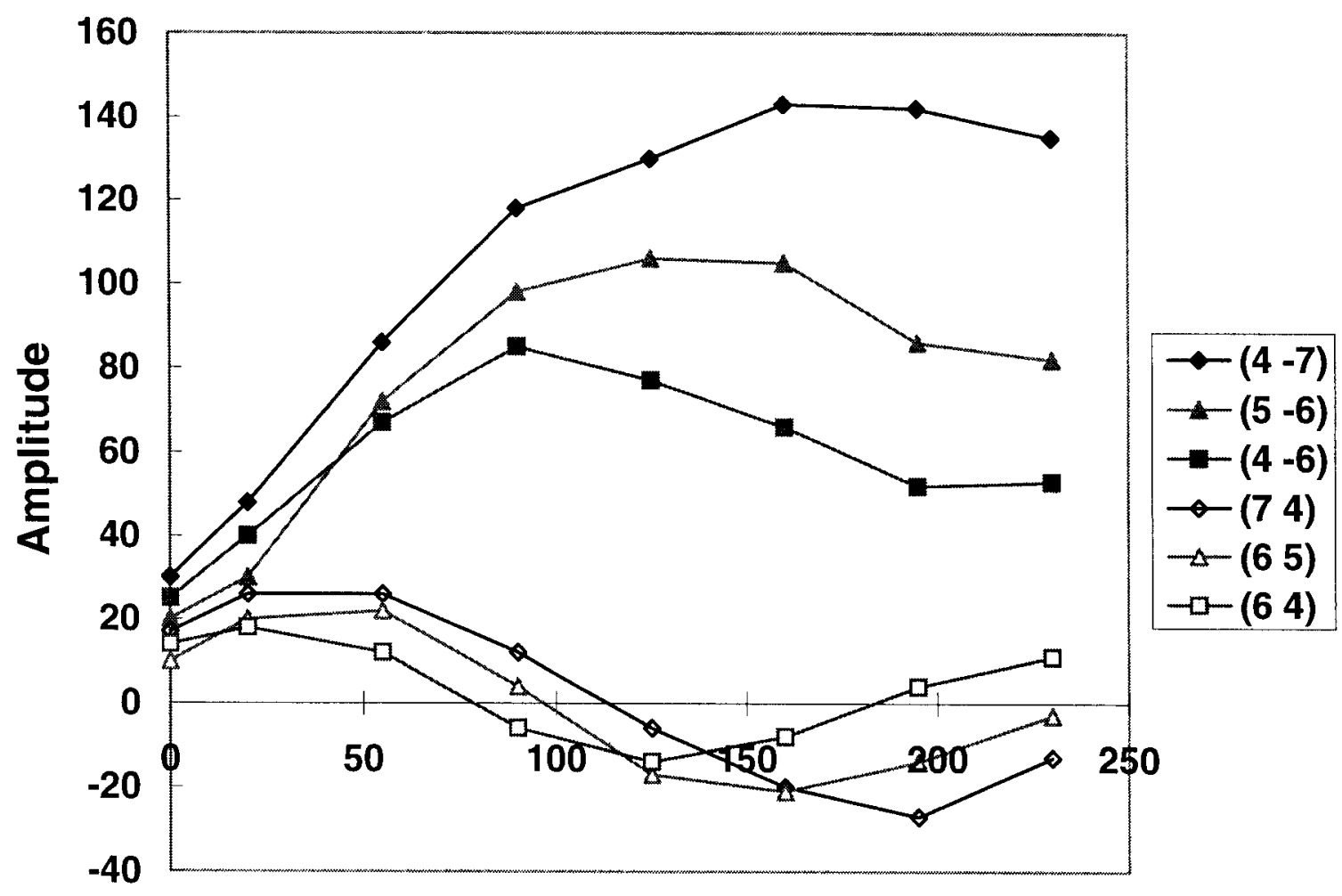

\section{Distance from edge of crystal $(\AA)$}

Fig. 6. Plots of image amplitudes of three pairs of symmetryrelated reflections from a crystal tilted by $2.5^{\circ}$ (the central row in Fig. 4). The threestrong reflections (with $\mathrm{k}<0$ ) are closeto the tilt axis and keep increasing with crystal thickness until the crystal is at least 100 $\AA$ thick. The three weaker reflections are all located perpendicularly to the tilt axis. They soon reach a maximum value, then decline until they have an amplitude of zero at the cross-over about $100 \AA$ in from the cell edge. As the crystal gets thicker further away from the edge, the amplitude increases again, but now with reversed phase.

TABLE 1. Amplitudes and phases for 4 of thestrongest among the 50 unique reflections from the crystal tilted by $1.1^{\text {o* }}$

\begin{tabular}{|c|c|c|c|c|c|c|c|c|c|c|c|c|c|c|}
\hline \multicolumn{2}{|c|}{ Index } & \multicolumn{6}{|c|}{ Amplitudes (distance from edge) } & & \multicolumn{6}{|c|}{ Phases (distance from edge) } \\
\hline $\mathrm{h}$ & $\bar{k}$ & $40 \AA$ & $80 \AA$ & $120 \AA$ & $160 \AA$ & $200 \AA$ & $240 \AA$ & & $40 \AA$ & $80 \AA$ & $120 \AA$ & $160 \AA$ & $200 \AA$ & $240 \AA$ \\
\hline $\begin{array}{l}4 \\
4 \\
6 \\
6 \\
5 \\
5 \\
6 \\
6 \\
4 \\
4 \\
7 \\
7 \\
6 \\
6\end{array}$ & $\begin{array}{r}-6 \\
6 \\
-4 \\
4 \\
-6 \\
6 \\
-5 \\
5 \\
-7 \\
7 \\
-4 \\
4 \\
-6 \\
6\end{array}$ & $\begin{array}{r}3,016 \\
3,131 \\
2,676 \\
2,401 \\
4,043 \\
3,174 \\
2,882 \\
3,296 \\
4,515 \\
3,544 \\
3,570 \\
2,646 \\
10,000 \\
7,211\end{array}$ & $\begin{array}{r}2,569 \\
1,744 \\
2,326 \\
1,313 \\
3,850 \\
1,955 \\
3,553 \\
2,048 \\
4,990 \\
2,243 \\
3,323 \\
1,327 \\
10,000 \\
3,630\end{array}$ & $\begin{array}{r}2,482 \\
1,232 \\
2,275 \\
614 \\
3,743 \\
615 \\
3,174 \\
1,120 \\
4,481 \\
1,108 \\
3,037 \\
613 \\
10,000 \\
1,415\end{array}$ & $\begin{array}{r}1,890 \\
848 \\
2,127 \\
0 \\
4,060 \\
0 \\
3,356 \\
0 \\
4,736 \\
504 \\
2,983 \\
576 \\
10,000 \\
1,196\end{array}$ & $\begin{array}{r}2,955 \\
470 \\
1,898 \\
284 \\
3,958 \\
665 \\
3,464 \\
282 \\
4,878 \\
381 \\
2,657 \\
546 \\
10,000 \\
2,153\end{array}$ & $\begin{array}{r}2,899 \\
219 \\
2,171 \\
445 \\
4,172 \\
308 \\
3,571 \\
720 \\
4,785 \\
0 \\
2,539 \\
1,149 \\
10,000 \\
2,453\end{array}$ & $\begin{array}{r}0 \\
0 \\
0 \\
0 \\
0 \\
180 \\
0 \\
180 \\
0 \\
180 \\
0 \\
180 \\
180 \\
180\end{array}$ & $\begin{array}{r}6 \\
-52 \\
-14 \\
19 \\
79 \\
-137 \\
68 \\
-100 \\
-51 \\
163 \\
51 \\
155 \\
-169 \\
162\end{array}$ & $\begin{array}{r}-31 \\
38 \\
-79 \\
-63 \\
29 \\
141 \\
4 \\
-174 \\
62 \\
95 \\
-21 \\
-161 \\
129 \\
-128\end{array}$ & $\begin{array}{r}5 \\
35 \\
-54 \\
-50 \\
65 \\
157 \\
42 \\
-175 \\
-52 \\
104 \\
21 \\
-107 \\
169 \\
-101\end{array}$ & $\begin{array}{r}15 \\
29 \\
-7 \\
104 \\
-44 \\
-40 \\
-59 \\
-45 \\
-20 \\
112 \\
31 \\
-50 \\
-171 \\
0\end{array}$ & $\begin{array}{r}12 \\
4 \\
3 \\
47 \\
-172 \\
5 \\
-173 \\
-167 \\
-5 \\
51 \\
-168 \\
4 \\
-5 \\
7\end{array}$ & $\begin{array}{r}-178 \\
109 \\
146 \\
-146 \\
85 \\
98 \\
47 \\
-110 \\
-77 \\
92 \\
-134 \\
66 \\
-29 \\
144\end{array}$ \\
\hline
\end{tabular}

*The column with phases in bold gives the correct phase values. The areas are all cut out parallel to the edge of the crystal and each area is $40 \AA$ by $300 \AA$. All amplitudes are scaled relative to the strongest of all reflections (66). Up to $120-160 \AA$ in from the edge of the crystal, the phases are close enough to the correct ones, at least for the strongest reflections closer to the tilt axis. Only the thickest areas (last two columns, 200-240 $\AA$ in from the crystal edge) were so distorted that they could not be reconstructed. The phase values listed here are those corresponding to the contrast of a positive print (black atoms), i.e., they are $180^{\circ}$ away from the X-ray phases.

strongest high-resolution reflections are shown in Table 1.

\section{Estimation of Crystal Thickness}

The crystal thickness was estimated from the ratio of amplitudes of symmetry-related reflections. The most accurate values are obtained at cross-overs of individual reflections; i.e., where the amplitude of a strong reflection off the tilt axis has fallen to zero. The high symmetry of this compound, space group P $4 / \mathrm{mbm}$ with projected symmetry $\mathrm{p} 4 \mathrm{~g}$, provided a great advantage for this study. There are al ways eight symmetry-rel ated 

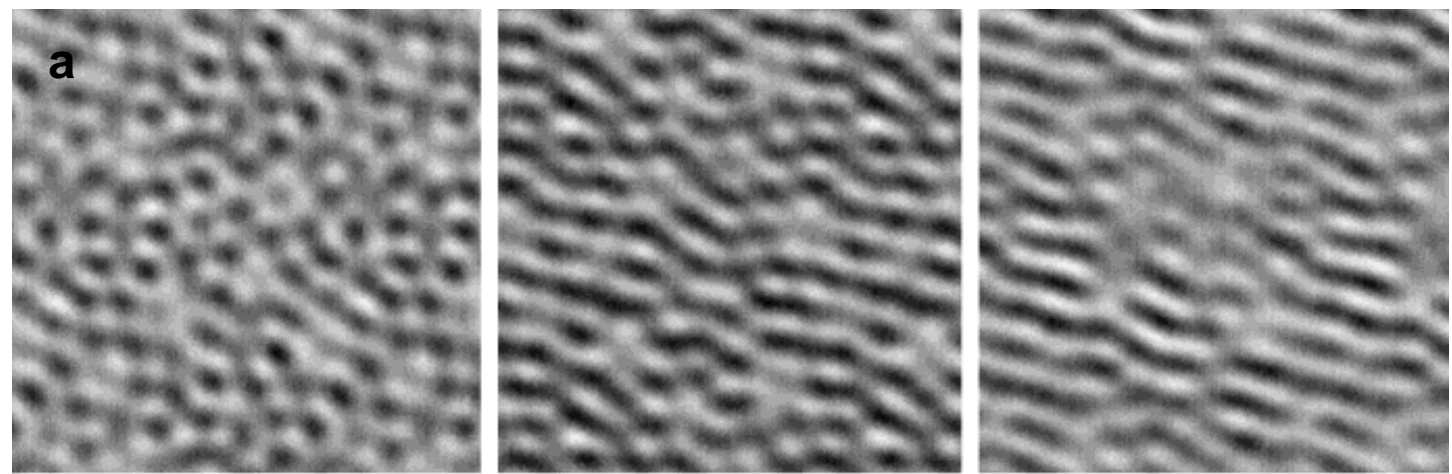

Distance
from edge
of crystal
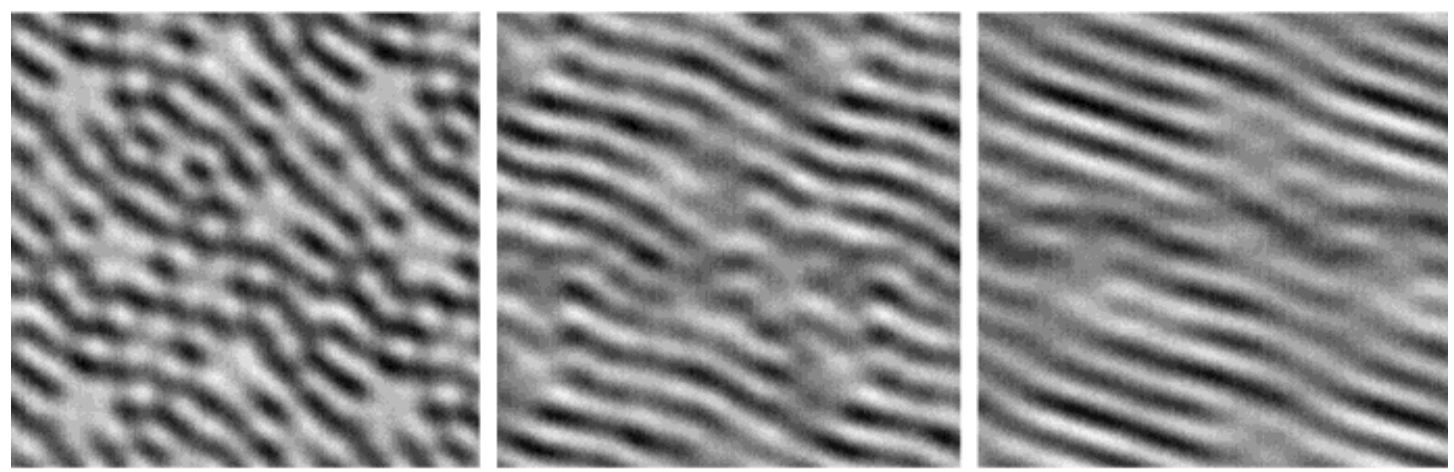

$0-120 \AA$

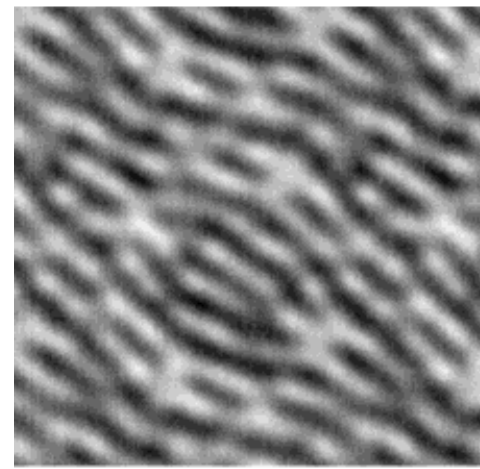

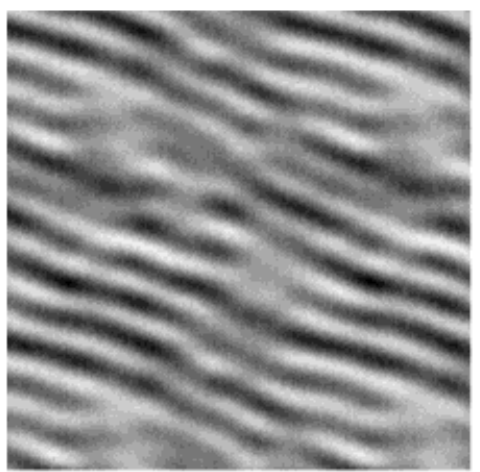

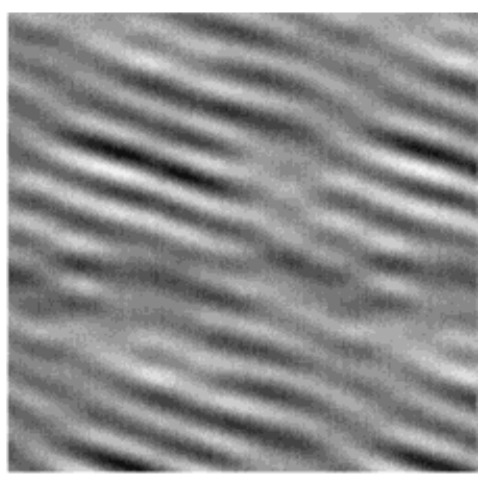

$4.1^{\circ}$ $2.5^{\circ}$

Tilt angle: $\quad 1.1^{\circ}$

Fig. 7. a: Lattice averaged areas, obtained from the Fourier transforms in Figure 4 . All reflections out to $2.5 \AA$ resolution are used, but no symmetry is applied. The structure, with its characteristic tunnels surrounded by 7 dark niobium atoms, can only be seen clearly in the top left corner, corresponding to the thinnest area and the smallest tilt angle.

reflections provide an excellent internal standard for quantitative work. Since all reflections in any one FT come from exactly the same area, we know that they must come from the same chemical composition, the same thickness, the same tilt angle and the same optical conditions (focus, astigmatism, etc.). This makes it much simpler to compare the effects of crystal tilt, than would have been the case with a crystal of lower symmetry. The cross-overs seen in Figures 5 and 6 must be caused by tilt effects and not for example multiple scattering or absorption, since their symmetry-related reflections, which are from equally thick regions, are not much different from what they were in the thin regions of the crystal. 

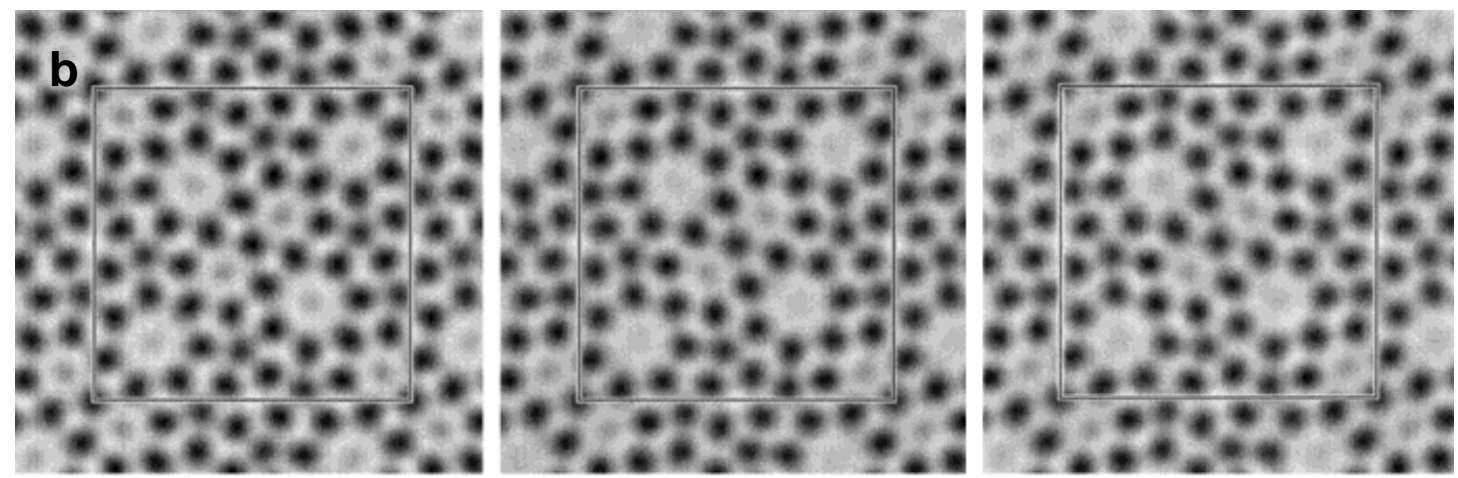

\section{Distance \\ from edge of crystal}
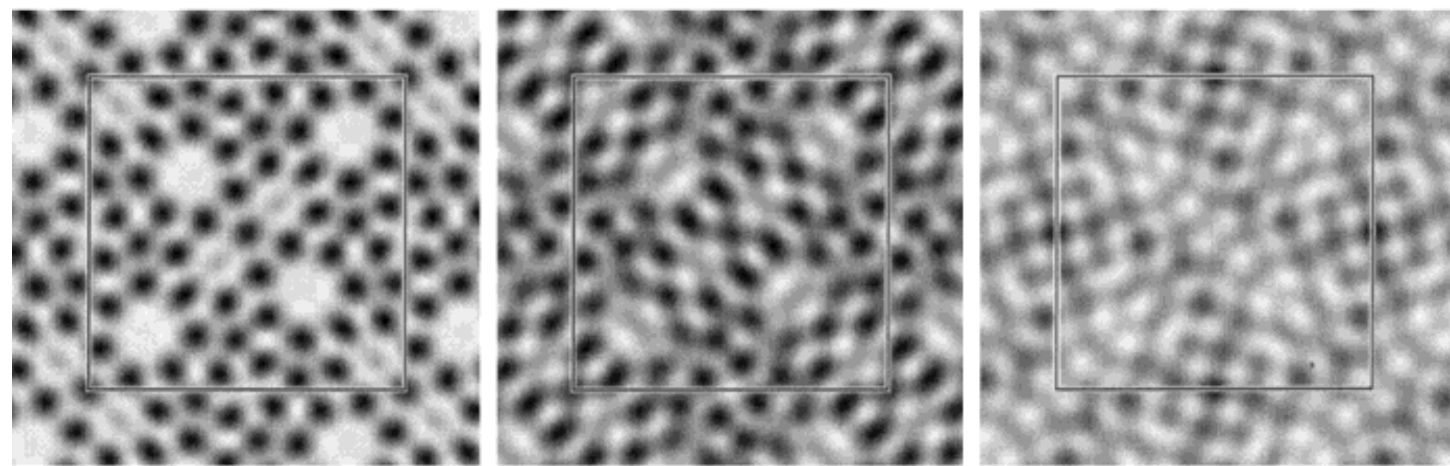

0-120 A
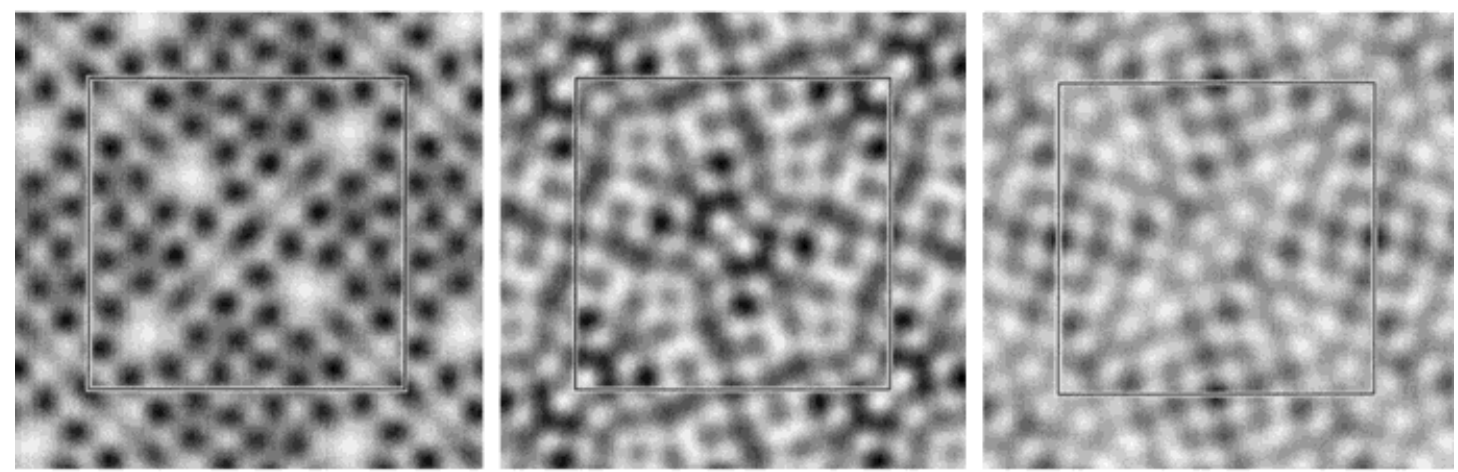

240-360 ̊̊

Tilt angle:

$1.1^{\circ}$

$2.5^{\circ}$

$4.1^{\circ}$

Fig. 7. b: Reconstructed projected potentials of the same areas as in Fig. 7a, but after imposing the correct crystallographic symmetry p4g. The unit cell $27.5 \AA$ by $27.5 \AA$ is indicated in each area. Now most of the areas give very clear structure images. Only when the crystal is both thick and highly tilted is it impossible to recognise the structure.

\section{Reconstruction of Projected Potential}

Projected potential maps were reconstructed by inverse FT of the extracted amplitudes and phases, before and after imposing the projected crystal symmetry p4g (Fig. 7). These maps were calculated from areas of increasing thickness on each of the differently tilted images. Each area was approximately $30 \mathrm{~nm}$ wide (parallel to the crystal edge) by $12 \mathrm{~nm}$ and cut out using the Edit tool in CRISP.

In every space group, the symmetry-related reflections all have the same ampl itude. However, the phase relations can be much more complicated than for $\mathrm{P} 4 /$ $\mathrm{mbm}$ in the $\mathrm{p} 4 \mathrm{~g}$ projection (see for example Hovmöller,
1981, 1997). The procedure of Crystallographic I mage Processing $(\mathrm{CIP})$ is to group symmetry-related reflections together, then average their amplitudes, and assign phases to the individual reflections according to the rules of the symmetry. In this projection, the plane group is $\mathrm{p} 4 \mathrm{~g}$ and the reflections in a group are (h k), $(h-k),(k h)$ and $(k-h)$. The phases must all be the same if $h+k=$ even. If $h+k=$ odd, then $(h-k)$ and $k-h)$ must have the same phases but differ by $180^{\circ}$ from the phases of ( $h k)$ and $(k h)$. Sincethe projection is centrosymmetric, all phases have to be $0^{\circ}$ or $180^{\circ}$. Thus there is only a choice between two possible sets of phases for each group of symmetry-related reflections. 
The amplitude and phase of each of the four symmetryrelated reflections are measured individual ly, but since they are coupled to each other, we can consider each reflection as one of four independent measurements of the same quantity.

\section{RESULTS}

Already a visual inspection of the HRTEM images of the crystal (Fig. 1) indicated that it was wedge-shaped and that the thickness increasd steadily and rather smoothly with distance from the edge. In all images, the thinnest part showed point-like features, corresponding to the niobium atoms. In the thicker regions, these points were smeared out intolines. This effect was more pronounced the more the crystals were tilted. In the HRTEM images that were tilted $3^{\circ}$ to $5^{\circ}$, the region where point-like atoms could be seen extended only 2 to $1 \mathrm{~nm}$, respectively, in from the edge.

The tilt angles that were calculated from the CBED patterns (Figs. 2 and 3) were rather close to the nominal tilt angles that had been read off the goniometer of the electron microscope. The nominal and measured angles differed by about $0.5^{\circ}$, and most of this discrepancy was due to a slight misalignment of the starting point of the tilt series; the nominally zerodegree tilt was in fact tilted by $0.4^{\circ}$. F or the quantitative analysis, we al ways used the tilt angles measured from the CBED patterns.

The FTs had significant diffraction maxima (reflections) out to 17 orders, corresponding to $0.16 \mathrm{~nm}$ resolution, which is also the point resolution of this microscope. Thestructure of $\mathrm{K}_{2} \mathrm{O} \cdot 7 \mathrm{Nb}_{2} \mathrm{O}_{5}$ has a characteristic ring of very strong reflections between 0.35 and $0.25 \mathrm{~nm}$ resolution (see Fig. 4).

The effects of crystal tilt and crystal thickness are seen in theFT (Fig. 4). The reflections on or very cl ose to the tilt axis are essentially unaffected by tilt and thickness. The further a reflection is from the tilt axis, the faster its amplitude is attenuated as the crystal gets thicker (going down in Fig. 4) or as it is tilted more (going right in Fig. 4). With a simpleapproximation, the attenuation $\mathrm{S}$ is described for weak phase objects by the $\operatorname{sinc}((\sin x) / x)$ function,

$$
\mathrm{S}\left(\mathrm{t}, \theta, \mathrm{u} \cdot \mathrm{r}^{0}\right)=\frac{\sin \left[\pi \mathrm{t}(\sin \theta) \mathrm{u} \cdot \mathrm{r}^{0}\right]}{\pi \mathrm{t}(\sin \theta) \mathrm{u} \cdot \mathrm{r}^{0}}
$$

(Zou, 1995) where $t$ is the crystal thickness, $\theta$ the tilt angle and $\mathrm{u}$ is the reciprocal vector of a reflection and $\mathrm{r}^{0}$ is a unit vector perpendicular to both the tilt axis and the incident beam. From this formula, it is predicted that the phases are unchanged for small tilts and thin crystals, but that they switch by $180^{\circ}$ at the point where the amplitude reaches zero. If the crystal is tilted slightly more or is slightly thicker, the reflection reappears after the cross-over has been reached, but now with reversed phase; i.e., with inverted contrast. That this indeed is what happens is most easily seen by looking at an oblique angle (with the eye close to the paper) al ong the set of lines in Figure 5 . Notice that when the lines continue on the other side of a crossover, all white lines turn into black and vice versa.

The simple behavior of the reflections as sinc functions is here confirmed experimentally. Only a few typical examples are shown in Figures 5 and 6 , but all reflections behaved in this way in all parts of the images.

\section{Determination of Crystal Thickness}

The crystal thickness $t$ at the cross-overs of any reflection can be calculated from (1), using the formula

$$
\mathrm{d}=\mathrm{t} \sin \theta
$$

since the first cross-over of a sinc function comes where $\mathrm{x}=\pi$.

If the reflection lies perpendicular to the tilt axis, then $\left(u \cdot r^{0}\right)^{-1}$ is equal to the d-value of that reflection. We see from Figure 6 that the cross-overs of reflections $(6,4)$ and $(6,5)$ and $(7,4)$ come at distances of 8,10 , and $11 \mathrm{~nm}$, respectively, in from the edge of the crystal, when the crystal is tilted by $2.5^{\circ}$. The values of $\left(u \cdot r^{0}\right)^{-1}$ for these three reflections are $0.38,0.35$, and $0.34 \mathrm{~nm}$, respectively and they are all nearly perpendicular to the tilt axis (see Fig. 4). From these values, the thickness $t$ can be estimated as $0.38 \mathrm{~nm} / \mathrm{sin} 2.5 \Rightarrow \mathrm{t}=8.7 \mathrm{~nm}$ at a distance of $80 \mathrm{~nm}$ from the edge, and $0.35 \mathrm{~nm} / \mathrm{sin}$ $2.5 \Rightarrow 8.1 \mathrm{~nm}$ at $10 \mathrm{~nm}$ in from the edge, and finally 0.34 $\mathrm{nm} / \mathrm{sin} 2.5 \Rightarrow \mathrm{t}=7.8 \mathrm{~nm}$ at a distance of $11 \mathrm{~nm}$ from the edge. From the average of these three cross-overs, the thickness is estimated to be about $8 \mathrm{~nm}$ at a distance of $10 \mathrm{~nm}$ in from the edge. There is a second cross-over at about $22 \mathrm{~nm}$ from the edge, corresponding to a thickness of $16 \mathrm{~nm}$. Similar plots were made from many reflections on all the images, tilted by various amounts. The resulting values all indicate that this crystal is wedge-shaped with an angle of cl ose to $45^{\circ}$ (see Fig. 8). There is a certain spread in the data, so the uncertainty in the estimate of crystal thickness by this method is about $\pm 15 \%$. One reason for this uncertainty may be that the crystal thickness is not always the same a certain distance from the edge. This causes the wavy appearance of the black lines of cross-overs seen in Figure 5.

\section{Reconstruction of Projected Potential From Tilted Crystals}

In order to solve a crystal structure, it is necessary to know the crystallographic structure-factor phase of about one strong, crystal lographical ly unique reflection per atom to be local ized. In the case of a crystal with one short (about $4 \AA$ ) unit cell axis, as in this case, the structure can be solved from a single projection. We do not expect to find the oxygen atoms or even the potassium atoms, so what we need is about one unique reflection per niobium atom. In the structure of $\mathrm{K}_{2} \mathrm{O} \cdot 7 \mathrm{Nb}_{2} \mathrm{O}_{5}$, there are eight unique niobi um atoms, so we expect to need correct phases for about the eight strongest unique reflections. Although the diffraction patterns in the FTs calculated from the images extends to $0.16 \mathrm{~nm}$, the strong reflections do not go further than about $0.25 \mathrm{~nm}$ resol ution. A common feature of this type of compound is that there is often a ring of strong diffraction maxima at 0.32 to $0.25 \mathrm{~nm}$ resolution. There are a total of 50 unique reflections to $0.25 \mathrm{~nm}$ resolution. If the resolution is cut down to $0.33 \mathrm{~nm}$, the structure cannot be recognized any longer, because several strong reflections in the range 0.25 to $0.32 \mathrm{~nm}$ resolution are then omitted. On the other hand, it is 
Fig. 8. The crystal is wedge-shaped with
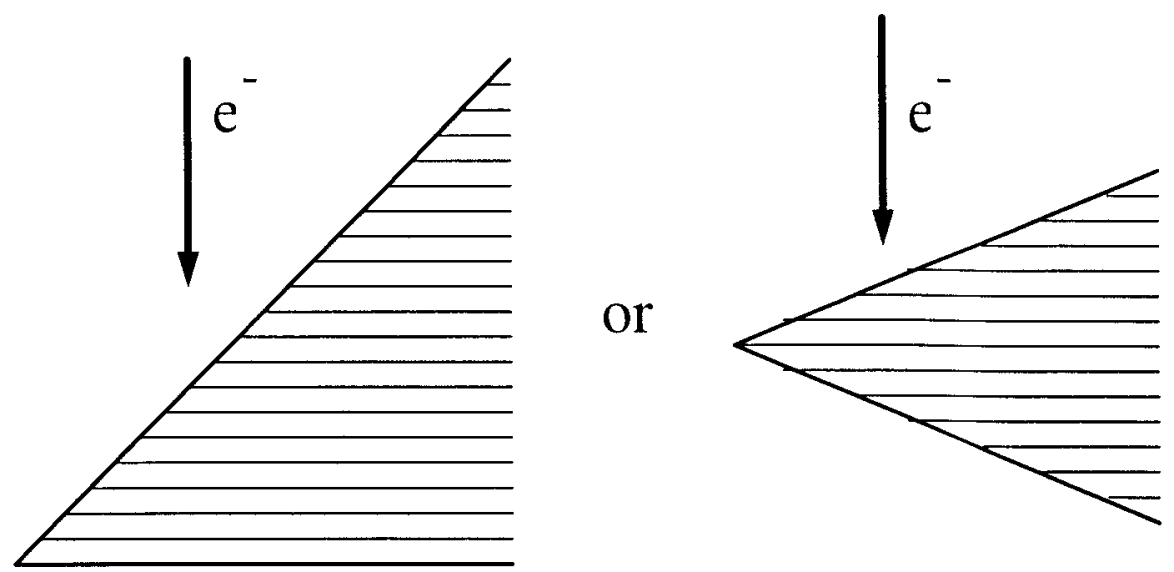
an angle close to $45^{\circ}$ !

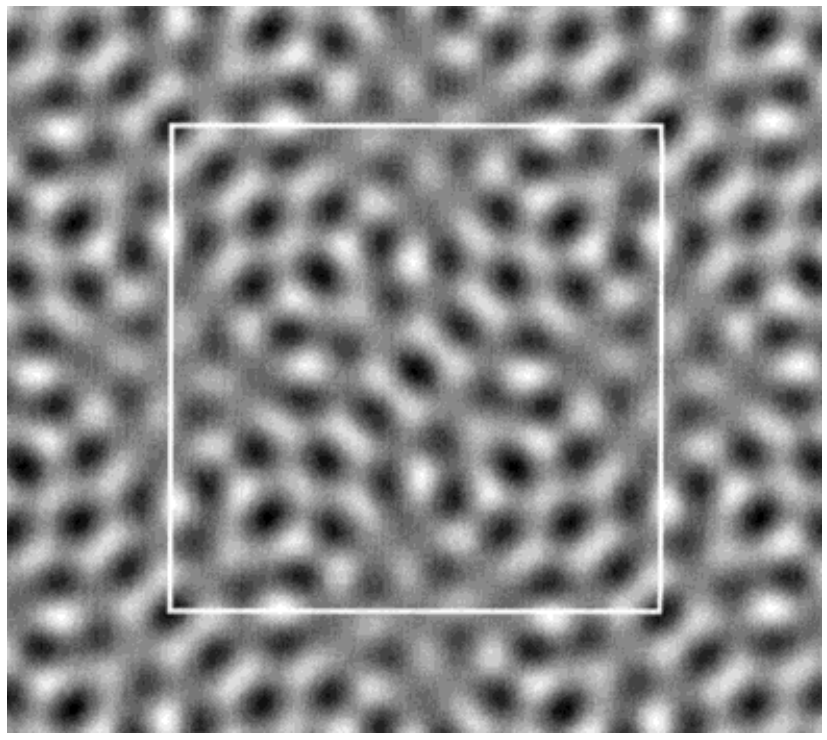

Fig. 9. Projected potential of $\mathrm{K}_{2} \mathrm{O} \cdot 7 \mathrm{Nb}_{2} \mathrm{O}_{5}$ reconstructed using only the 5 strongest unique reflections and their symmetry-related reflections in p4g. All the $\mathrm{Nb}$ atoms are seen at their correct locations. The $\mathrm{K}$ atoms in the 7- and 6-fold rings are unduly strong, but otherwise the map is very similar to the ones where all reflections are included (see Fig. 7b).

easy to recognize the structure from as few as the five(!) strongest reflections within $0.25 \mathrm{~nm}$ resolution (see $\mathrm{Fig}$. 9). These were the reflections (0 8), (2 8) (3 7), (3 8), and (6 6). If any one of these five strongest reflections was deleted or its phase reversed, the structure could no longer be recognized.

As shown above, when a crystal is tilted, the amplitudes and phases of the reflections are affected. For small tilt angles and relatively thin crystals, only the amplitudes and not the phases are changed. The structure can easily be recognized within this region. When the tilt and thickness are so large that phases start to be reversed, the situation rapidly becomes very difficult. However, when the symmetry is high (3-, 4- or 6-fold) as in this case, it is possible to obtain the correct structure also after phases have started to reverse, by imposing the correct crystal symmetry. (It is assumed that it has been possible to determine the symmetry correctly. If not, imposing a wrong symmetry will only make things worse.)

For thin areas and small tilt angles, the amplitudes of symmetry-related reflections are quite similar, with $R_{\text {sym }}$ of less than $20 \%$. $R_{\text {sym }}$ is defined by

$$
\mathrm{R}_{\text {sym }}=\sum_{\mathrm{i}} \frac{|| \mathrm{F}_{\mathrm{i}}(\mathrm{hkl})|-\langle|\mathrm{F}(\mathrm{hkl})|\rangle|}{\langle|\mathrm{F}(\mathrm{hkl})|\rangle},
$$

where $\langle\mathrm{F}(\mathrm{hkl})\rangle$ is the average amplitude of all reflections symmetry-related to $(\mathrm{hkl})$, and $\mathrm{F}_{\mathrm{i}}(\mathrm{hkl})$ stands for each individual of these symmetry-related reflections. The phases are very good; the amplitude-weighted phase residual is under $20^{\circ}$; i.e., the phases are close to one of the two allowed phase values, $0^{\circ}$ and $180^{\circ}$, and the phases of four symmetry-related reflections also follow the above-mentioned rules of the symmetry. As the crystals get thicker and/or more tilted, first the amplitudes and then the phases are changed. The effect is stronger the further away from the tilt axis the reflection is. This can be followed qualitatively by looking at the amplitudes in the FT's in Figure 4, and numerically for four strong fairly high-resolution reflections, in Table 1. At higher tilts and for thicker crystals, some reflections start to get wrong phases. However, it is still possible to determine the correct phases for the whole group, since the reflections close to the tilt axis are not much affected, and (because of the high symmetry of this crystal) there are always one or two equivalent reflections close to the tilt axis. The reflections close to the tilt axis have the further advantage that their amplitudes are rather unaffected by crystal tilt. When there are conflicting indications of phase values for a group of symmetry-related reflections, CRISP decides which phases to assign based on a scheme where the reflections are amplitude weighted. The amplitudes of reflections near the tilt axis remain strong. When the phase values of a group of symmetryrelated reflections are to be determined, these reflections will dominate the outcome. Sincethe phases of the reflections close to the tilt axis are essentially correct, the whol e group of symmetry-related reflections will be given correct phases.

It is possible to reconstruct an interpretable projecti on map even for a crystal of about 15-20 nm thickness that has been tilted by $2.5^{\circ}$ (the central map in Fig. 7). 
TheFT of this area is shown in the center of Figure 4. It is evident that the amplitudes of more than half the reflections are approaching zero. Furthermore, all the strong reflections around $(6,6)$ have reversed phases at this tilt and thickness, as can be seen from Figure 5. The reason why the structure can be reconstructed even from such a distorted image is that there are enough well-estimated reflections in the band of reflections around the tilt axis. Due to the high symmetry, at least one of the reflections from each group of symmetryrelated reflections is close to the tilt axis. Those reflections have the largest amplitudes in each group of symmetry-related reflections and their phases are still close to the correct ones. Since these are the strongest reflections in each group, they will force the whole group to get correct phase values. This is enough to reconstruct all unique reflections, with virtually no phase errors, and from this an interpretable map is obtained by inverse FT.

If the crystal is too thick and/or too much tilted, it is not possible to reconstruct the structure (see the three maps in the bottom right of Fig. 7). The reason is obvious from looking at the FT's (Fig. 4); there are too few well-measured reflections in the very narrow band around the tilt axis. Even after applying the high p4g symmetry, many groups of strong reflections had no member close enough to the tilt axis, and thus the reflections could not be correctly phased.

\section{DISCUSSION}

Probably the most surprising result of this study is that such a transparent crystal as the one used here has a wedge angle of close to $45^{\circ}$ and thus rapidly becomes quite thick. It must be expected that other crystals of approximately similar chemical composition (metal oxides) and similar thickness are equally electron transparent.

Another remarkable conclusion is that it is possible to reconstruct the crystal structure from HRTEM images from areas with a thickness of 10-20 nm.

The experimentally quantified effects of crystal tilt and thickness described here (Fig. 6 and Table 1) follow closely what was theoretically predicted (Zou, 1995). The smooth and well-behaved changes of amplitudes and stability of phases over a wide range of thickness and tilt may explain why crystallographic image processing has been so successful in solving unknown crystal structures from HRTEM images.

It should be stressed that the reason why we chose a crystal with so high a symmetry for this study was that we wanted the "internal standards" of symmetryrelated reflections, which we know haveidentical structure factors, in order to obtain quantitative results.

It was possible to reconstruct a very cl ear and correct projected potential map from areas of about $20 \mathrm{~nm}$ thickness, tilted by $2.5^{\circ}$. F or crystals with lower symmetry, it will not necessarily be possible to reconstruct the structure from such thick and highly tilted crystals. A somewhat modified approach must betaken for crystals with lower symmetry. If the effects of tilt are not so large that the phases of some strong high-resolution reflections have been reversed, then it may suffice to replace the amplitudes from the HRTEM images by amplitudes determined from SAED patterns. An even more elaborate scheme could be to locate the tilt axis, then quantify the thickness and tilt angle and finally correct the amplitudes and phases of those reflections, by multiplying with the inverse of formula (1). However, in practice, the simplest and best procedure is to align the crystal to within a fraction of a degree, and then to process the thinnest area near the edge of a transparent crystal. Typically the amplitudes are correct to within $20 \%$ and the phases to within $20-30^{\circ}$ in such cases. If the crystal has a 2 -fol d axis parallel to the electron beam, then the projection is centrosymmetric, and the phases can befurther improved by setting them to the nearest of $0^{\circ}$ and $180^{\circ}$. The defocus must of course be checked by inspecting the FT and if the image turns out not to have been taken very cl ose to Scherzer defocus, a correction must be applied, as described (Zou et al., 1996). If the image is taken along a short unit cell dimension $(<5 \AA)$ and the resolution of the electron microscope is high enough for resolving the features of interest (about $0.25 \mathrm{~nm}$ resolution for metals in oxides and about $0.17 \mathrm{~nm}$ for alloys), the structure can be solved from a single projection. If the shortest crystal axis is larger than about $6 \AA$, it is necessary to make a 3D structure determination, by combining views along several zone axes.

The accuracy of co-ordinates for the metal atoms in this structure, after reconstruction by crystallographic image processing, is 0.1 to $0.2 \AA$. This is sufficiently close to the correct values to serve as a starting point for the final step in a structure determination-the refinement (Weirich et al., 1996).

The method presented here can be used routinely for thickness determination, such that any comparisons with image simulations then are done with experimentally determined, rather than guessed, thickness values. This could be very useful for quantitative comparisons between experimental and simulated images, a field that is just emerging (Kilaas, 1997).

\section{CONCLUSION}

Crystal structures can be solved from HRTEM images because the crystallographic structure factors can be determined with sufficient accuracy from the amplitudes and phases of the FT of HRTEM images. A structure can be deduced directly from an HRTEM image only if the experimental conditions are within a very narrow range of optimal. With image processing, it is possible to extend the range of experimental conditions considerably. This greatly facilitates structure determination from HRTEM images.

It is now possible to determine all the parametersdefocus, astigmatism, tilt, and thickness-experimentally, so there is no longer any need to simulate a set of images with guessed focus and thickness values. Since the thickness can be experimentally determined, it is possible to say with certainty which (if any) parts of a crystal are sufficiently thin for the weak phase object approximation to be satisfied.

The conclusions of the present investigation are very different from those of J ansen et al. (1998) who claim that "the kinematical diffraction theory can only be applied for thicknesses up to about $2 \mathrm{~nm}$ for strong scatterers." The reasons for these highly divergent opinions need to be found out by future collaborative efforts, where the different methods are applied on the same samples. 


\section{ACKNOWLEDGMENTS}

The crystals of $\mathrm{K}_{2} \mathrm{O} \cdot 7 \mathrm{Nb}_{2} \mathrm{O}_{5}$ were kindly provided by Dr. Q.Z. Wu, Institute of Physics, Chinese Academy of Sciences, Beijing. The HRTEM images were taken at the National Center for Electron Microscopy in Lund.

\section{REFERENCES}

Bhide V, Gasparin M. 1979. A new GTB-type thallium niobate. Acta Cryst B35:1318-1321.

Downing KH, Meisheng Hu, Wenk H-R, O'Keefe MA. 1990. Resolution of oxygen atoms in staurolite by three-dimensional transmission electron microscopy. Nature 348:525-528.

Erickson HP, Klug A. 1971. Measurement and compensation of defocusing and aberrations by Fourier processing of electron micrographs. Phil Trans R Soc Lond B261:105-118.

$\mathrm{H}$ an SF, Fan HF, Li FH. 1986. Image processing in high-resolution electron microscopy using the direct method. II. I mage deconvolution. Acta Cryst A42:353-356.

Hovmöller S. 1981. Origin specification. In part published in Pamphlet Series issued by the Education Committee of the Int Union Crystallogr 9:1-11.

Hovmöller S. 1992. CRISP: Crystallographic image processing on a personal computer. Ultramicroscopy 40:121-135.

Hovmöller S. 1997. The effects of symmetry in real and reciprocal space. In: Dorset DL, Hovmöller S, Zou XD, editors. Electron crystallography. Nato ASI Series E. Kluwer Academic Publishers. 347:139-150.

Hovmöller S, Sjögren A, Farrants G, Sundberg M, Marinder BO. 1984. Accurate atomic positions from el ectron microscopy. Nature 311:238241.

Hu J, Li FH, Fan HF. 1992. Crystal structure determination of $\mathrm{K}_{2} \mathrm{O} \cdot 7 \mathrm{~N} \mathrm{~b}_{2} \mathrm{O}_{5}$ by combining high-resolution electron microscopy and electron diffraction. Ultramicroscopy 41:387-397.

J ansen J, Tang D, Zandbergen HW, Schenk H. 1998. MSLS, a least-squares procedure for accurate crystal structure refinement from dynamical electron diffraction patterns. Acta Cryst A54:91101.
Kilaas R. 1997. Structure refinement through matching of experimental and simulated HRTEM images. In: Dorset DL, Hovmöller S, Zou $X D$, editors. Electron crystallography. NatoASI Series E. Dordrecht: Kluwer Academic Publishers 347:247-260.

Li FH. 1998. Image processing based on the combination of highresolution electron microscopy and electron diffraction. Microsc Res Tech 40:86-100.

O'Keefe MA, Radmilovic V. 1993. The effects of small crystal tilts on dynamical scattering: Why simulated images are thinner than experimental ones. Cincinatti, $\mathrm{OH}$ : 51st Ann Proc Microsc Soc Am p 980-981.

Saxton WO, Smith DJ . 1985. The determination of atomic positions in high-resolution electron micrographs. Ultramicroscopy 18:39-48.

Spence J CH, Cowley J M. 1995. Electron diffraction. In: Wilson AJ C, editor. International tables for crystallography, Vol C. Dordrecht Kluwer Academic Publishers. p 371.

Unwin PNT, Henderson R. 1975. Molecular structure determination by electron microscopy of unstained crystalline specimens. J Mol Biol 94:425-440.

Van Dyck D, Lichte H, Van der Mast KD. 1996. Sub-Ångström structure characterisation: the brite-euram route towards one Ångström. Ultramicroscopy 64:1-15.

Wang DN, Hovmöller S, Kihlborg L, Sundberg M. 1988. Structure determination and correction for distortions in HREM by crystallographic image processing. Ultramicroscopy 25:303-316.

Weirich T, Ramlau R, Simon A, Zou XD, Hovmöller S. 1996. A crystal structure determined to $0.02 \AA$ accuracy by electron microscopy. Nature 382:144-146.

Zou XD. 1995. Electron crystallography of inorganic structures: theory and practice. Chem Commun 5, Stockholm University, Doctoral thesis.

Zou XD, Ferrow EA, Hovmöller S. 1995. Correcting for crystal tilt in HRTEM images of minerals: the case of orthopyroxene. Phys Chem Minerals 22:89-92.

Zou XD, Sundberg M, Larine M, Hovmöller S. 1996. Structure projection retrieval by image processing of HREM images taken under non-optimal defocus conditions. Ultramicroscopy 62:103-121. 